\title{
Lattice Fluid Dynamics from Perfect Discretizations of Continuum Flows *
}

\author{
E. Katz and U.-J. Wiese \\ Center for Theoretical Physics, \\ Laboratory for Nuclear Science, and Department of Physics \\ Massachusetts Institute of Technology (MIT) \\ Cambridge, Massachusetts 02139, U.S.A. \\ MIT Preprint, CTP 2423
}

February 22, 2019

\begin{abstract}
We use renormalization group methods to derive equations of motion for large scale variables in fluid dynamics. The large scale variables are averages of the underlying continuum variables over cubic volumes, and naturally live on a lattice. The resulting lattice dynamics represents a perfect discretization of continuum physics, i.e. grid artifacts are completely eliminated. Perfect equations of motion are derived for static, slow flows of incompressible, viscous fluids. For Hagen-Poiseuille flow in a channel with square cross section the equations reduce to a perfect discretization of the Poisson equation for the velocity field with Dirichlet boundary conditions. The perfect large scale Poisson equation is used in a numerical simulation, and is shown to represent the continuum flow exactly. For non-square cross sections we use a numerical iterative procedure to derive flow equations that are approximately perfect.
\end{abstract}

${ }^{*}$ This work is supported in part by funds provided by the U.S. Department of Energy (D.O.E.) under cooperative research agreement DE-FC02-94ER40818. 


\section{Introduction}

Problems of fluid flow are of great practical importance in such areas as mechanical engineering and meteorology. A full theoretical understanding of fluid flow is very difficult because of the complex dynamics. In particular, in the regime of fully developed turbulence we face a dynamical system with fluctuations on all length scales. The small scale structures influence the dynamics at larger scales in a way that is hard to control analytically. Numerical simulations are therefore an essential tool in fluid dynamics. However, with present day computers a full simulation of realistic problems from first principles is beyond reach. Already simple model problems exhaust the biggest machines available. Hence, in practical applications one makes additional assumptions, especially about the small scale dynamics, which are difficult to justify theoretically, and which involve free parameters. In fact, the primary systematic error in numerical simulations of fluid flow is due to the finite grid size that is necessary to discretize the continuum Navier-Stokes equations.

The main purpose of this paper is to point out that perfect discretizations, i.e. ones that are completely free of grid artifacts, exist and can be constructed explicitly. The existence of perfect discretizations is a direct consequence of Wilson's renormalization group theory [1]. The idea of the renormalization group is to deal with the fluctuations at different length scales step by step in scale. In this way the influence of small scale fluctuations on the large scale dynamics can, at least in principle, be controlled exactly. Recently, in the context of lattice quantum field theories so-called perfect actions have been constructed for various models. Originally, Hasenfratz and Niedermayer iterated a renormalization group transformation to obtain a perfect discretization for the 2-dimensional $O(3)$ nonlinear $\sigma$-model, and they demonstrated that grid artifacts were eliminated even on coarse lattices [2]. The same method has been applied to lattice fermions [3], 4], to 4-dimensional pure $S U(3)$ gauge theory [5] and to full QCD [6]. Here we use similar methods in classical physics. In principle, the renormalization group can be used to derive perfect discretizations for any differential equation. Examples of great practical importance are the Laplace and Poisson equations, Maxwell's equations and the Navier-Stokes equation. A perfect discretization of the Laplace equation was obtained by Bell and Wilson in an early study of scalar lattice field theory [8]. The perfect action for a classical gauge field - and hence a perfect discretization of Maxwell's equations has been constructed in refs. [5, 6]. In this work we concentrate on the Navier-Stokes equations. Here we do not treat the problem in its full complexity yet. Specifically, we restrict ourselves to incompressible fluids and we assume static, slow flows.

The first step in our program is to define large scale lattice variables from the underlying continuum fields. In the case of the Navier-Stokes equations these are the velocity and pressure fields. We average the continuum pressure over a cube of side length $a$ to define the large scale pressure variables, which then naturally live on a lattice of spacing $a$ consisting of the cube centers. Similarly, the velocity 
field is averaged over the interface between two neighboring cubes, and hence the corresponding lattice field lives on the links connecting neighboring lattice points. This construction ensures that the continuity equation maintains a simple form on the lattice. By construction we know exactly how the large scale variables are related to the continuum fields. The goal of the next step is to derive equations of motion for the large scale fields. Solving these equations yields exact results for the averaged continuum quantities. This is in contrast to standard discretization procedures, where the lattice field represents an approximation of the continuum field at the same point. The approximation becomes exact only in the continuum limit $a \rightarrow 0$, because the value of the field at a specific point is influenced by the dynamics at arbitrarily small scales. A perfect discretization, on the other hand, ensures exact results for averaged continuum quantities already at finite $a$. Still, the values of the continuum fields can be reconstructed from the lattice data by using so-called perfect fields.

The second step is to derive exact equations of motion for the lattice fields. One possibility to do that is to iterate a discrete renormalization group transformation. This is done by starting on a very fine lattice (in fact, at the end the lattice spacing of the fine lattice is sent to zero), and then performing an infinite sequence of blocking steps, in which the lattice fields are averaged over blocks, and effective equations of motion for the block variables are derived. Here we proceed differently by blocking directly from the continuum. The result is identical to the discrete, iterative procedure, but the derivation is more transparent. Wilson was the first to discover this technique [7], which has not been very well known until recently. For example, blocking from the continuum was also used in the derivation of the QCD perfect action [6]. In contrast to quantum field theory there is no action for the full NavierStokes problem. Still, blocking from the continuum can be directly applied to the equations of motion. The constraint that identifies the large scale lattice variables with the averaged continuum fields is implemented through a Lagrange multiplier. Solving the equations for the continuum variables in terms of the lattice fields and plugging the result back into the constraint equations leads to the equations for the large scale variables.

For numerical applications it is essential that the lattice equations of motion are as local as possible. In this paper we distinguish three types of locality: ultralocal, local and nonlocal. A lattice equation is called ultralocal if it couples the lattice field at a given site to a finite number of neighboring sites only. Consider, for example, the 1-dimensional Laplace equation

$$
\frac{d^{2} f(x)}{d x^{2}}=0
$$

Its standard lattice discretization

$$
\frac{1}{a^{2}}[f(x+a)-2 f(x)+f(x-a)]=0
$$


is ultralocal because it couples the field at the point $x$ to its nearest neighbors at $x+a$ and $x-a$ only. In general, a perfect discretization will not lead to an ultralocal coupling. The couplings to fields at distant sites are non-zero, but exponentially suppressed. In that case we say that the lattice equation is local. By optimizing the parameters of the renormalization group transformation one can control the strength of the exponential decay. It turns out that the parameters can be chosen such that the perfect equations become ultralocal in one dimension. In practice, even in higher dimensions this leads to extremely local perfect lattice equations, whose exponentially suppressed couplings to distant neighbors can safely be neglected. In particular, as it will become clear later, even the ultralocal eq.(1.2) turns out to be perfect. This would not be the case for the common approach to discretization problems, where one interprets lattice derivatives as approximations of continuum derivatives at the same point. Still, even then one can find a perfect discretization. Using Fourier transforms it is easy to show that a smooth function $f(x)$ can be reconstructed from its values $f(n a)$ on a lattice by

$$
f(x)=\sum_{n \in \mathbf{Z}} f(n a) \frac{\sin \pi(x / a-n)}{\pi(x / a-n)} .
$$

Here we have assumed that $f(x)$ is infinitely differentiable and that its Fourier transform exists. The above equation yields

$$
\frac{d^{2} f(m a)}{d x^{2}}=-\frac{1}{a^{2}}\left[\frac{\pi^{2}}{3} f(0)+2 \sum_{n \in \mathbf{Z} \backslash\{m\}} f(n a) \frac{(-1)^{n-m}}{(n-m)^{2}}\right] .
$$

This exactly represents the continuum second derivative of the function at the lattice point ma. However, now the lattice derivative comprises terms at arbitrarily large distances, and their contributions are suppressed only power-like. This is what we call a nonlocal coupling. In this case the contributions from large distances cannot be neglected and therefore this equation is not of practical use. In contrast, the advantage of using block averaged fields is that their perfect discretizations are local.

As opposed to previous applications of perfect discretizations, which dealt with infinite volumes or finite systems with periodic boundary conditions, the incorporation of more general boundary conditions is essential in fluid dynamics. While it is straightforward to implement periodic boundary conditions, it is more difficult to treat fixed boundary conditions, which occur in typical fluid dynamics applications. Still, it turns out that fixed boundary conditions can be represented exactly in the perfect lattice equations. Here we restrict ourselves to channels whose cross section is a rectangular polygon. Arbitrarily curved boundaries can in principle be handled using curvelinear coordinates, but we do not elaborate on this issue here.

The paper is organized as follows. In section 2 we introduce the large scale lattice pressure and velocity variables by blocking from the continuum, and we investigate 
their behavior under discrete renormalization group transformations. Section 3 contains the derivation of perfect equations of motion for Hagen-Poiseuille flow. The parameter in the renormalization group transformation is optimized for ultralocality in two dimensions. We verify explicitly that also in three dimensions continuum physics is exactly reproduced. In section 4 we show analytically that the equations of motion indeed satisfy a factor 2 renormalization group step. Section 5 presents results of a numerical calculation of the lattice fields. In section 6 we construct the perfect velocity field that allows reconstruction of the continuum field from the lattice data. Section 7 deals with static, slow flows in general. We derive the perfect lattice version of the corresponding Navier-Stokes equations. In section 8 we present a perturbative method of introducing the non-linear term to equation of motion. Finally, section 9 contains concluding remarks on how to extend our construction to the full Navier-Stokes equations and to arbitrarily shaped boundaries. We also speculate about applications of perfect discretizations to numerical simulations of turbulent flows.

\section{Large scale lattice variables}

The continuum Navier-Stokes equations are formulated in terms of pressure and velocity variables. Here we consider static flows in $d$ dimensions, and hence pressure $p(y)$ and velocity $v_{i}(y)$ with $i \in\{1,2, \ldots, d\}$ are functions of the position vector $y$ only. For incompressible fluids the velocity field obeys the continuity equation

$$
\partial_{i} v_{i}(y)=0
$$

To define the large scale lattice pressure variables $P_{x}$ the continuum pressure field $p(y)$ is averaged over a $d$-dimensional cube $c_{x}$ of side length $a$ centered at $x$,

$$
P_{x}=\frac{1}{a^{d}} \int_{c_{x}} d^{d} y p(y)
$$

The variables $P_{x}$ naturally live on a lattice of spacing $a$ formed by the cube centers $x$. Similarly, for the velocity field we define

$$
V_{i, x}=\frac{1}{a^{d-1}} \int_{f_{i, x}} d^{d-1} y v_{i}(y)
$$

where $f_{i, x}$ is the $(d-1)$-dimensional face separating the cubes $c_{x-a \hat{i} / 2}$ and $c_{x+a \hat{i} / 2}$. Note that $\hat{i}$ is the unit vector in the $i$-direction. It is natural to associate the velocity variables with the links connecting neighboring lattice sites $x-a \hat{i} / 2$ and $x+a \hat{i} / 2$. Now the $i$-component of $x$ is a half-integer multiple of the lattice spacing. The lattice velocity variables can be interpreted as volume flow rates per cross sectional area of the faces separating neighboring cubes. This definition of the lattice velocity 
field is natural because the lattice continuity equation then assumes an ultralocal form. It is simply given by

$$
\begin{aligned}
\delta V_{x} & =\sum_{i}\left[V_{i, x+a \hat{i} / 2}-V_{x-a \hat{i} / 2}\right]=\frac{1}{a^{d-1}} \sum_{i}\left[\int_{f_{i, x+a \hat{i} / 2}} d^{d-1} y v_{i}(y)-\int_{f_{i, x-a \hat{i} / 2}} d^{d-1} y v_{i}(y)\right] \\
& =\frac{1}{a^{d-1}} \int_{\partial c_{x}} d^{d-1} \sigma_{i} v_{i}(y)=\frac{1}{a^{d-1}} \int_{c_{x}} d^{d} y \partial_{i} v_{i}(y)=0 .
\end{aligned}
$$

Here we have used Gauss' law together with the continuum continuity equation. The definition of lattice variables by blocking from the continuum can be viewed as a renormalization group transformation with an infinite blocking factor. In fact, the same result could be obtained by iterating block factor 2 transformations starting from an arbitrarily fine lattice. To identify the corresponding factor 2 transformation we now block from the continuum to a lattice of twice the lattice spacing $a^{\prime}=2 a$. The continuum pressure variables are then integrated over cubes $c_{x^{\prime}}^{\prime}$ of side length $a^{\prime}$ which contain $2^{d}$ original cubes $c_{x}$. Hence, the resulting pressure variable

$$
P_{x^{\prime}}^{\prime}=\frac{1}{a^{\prime d}} \int_{c_{x^{\prime}}^{\prime}} d^{d} y p(y)=\frac{1}{2^{d}} \sum_{x \in x^{\prime}} \frac{1}{a^{d}} \int_{c_{x}} d^{d} y p(y)=\frac{1}{2^{d}} \sum_{x \in x^{\prime}} P_{x}
$$

on the coarse lattice is a block average of the pressure variables on the fine lattice. We use $x \in x^{\prime}$ to denote that the cube $c_{x}$ belongs to the block $c_{x^{\prime}}^{\prime}$. The corresponding geometry is illustrated in figure 2. Similarly, for the velocity field we have

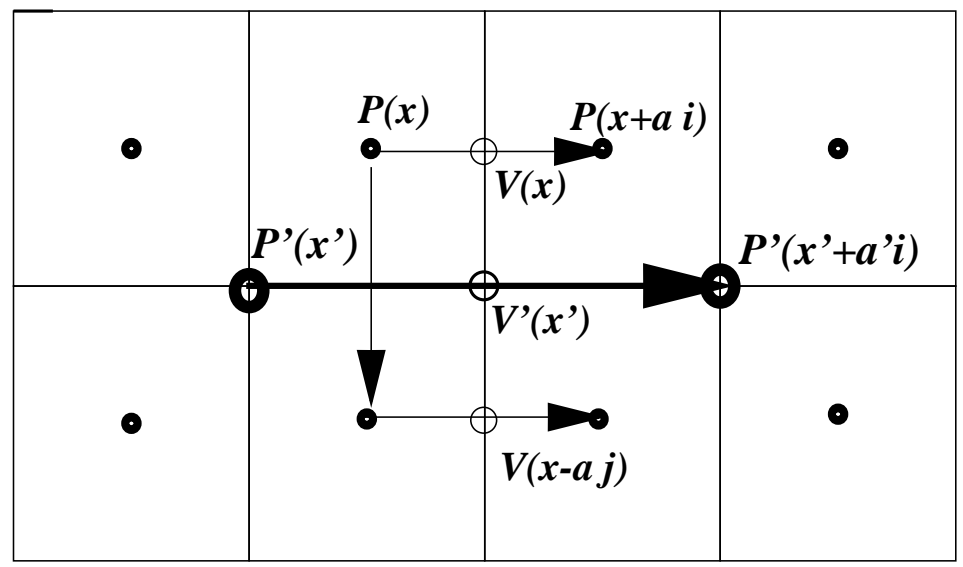

Figure 1: The geometry of the block factor 2 renormalization group transformation.

$$
\begin{aligned}
V_{i, x^{\prime}}^{\prime} & =\frac{1}{a^{\prime d-1}} \int_{f_{i, x^{\prime}}^{\prime}} d^{d-1} y v_{i}(y) \\
& =\frac{1}{2^{d-1}} \sum_{x \in x^{\prime}} \frac{1}{a^{d-1}} \int_{f_{i, x}} d^{d-1} y v_{i}(y)=\frac{1}{2^{d-1}} \sum_{x \in x^{\prime}} V_{i, x} .
\end{aligned}
$$

Here $x \in x^{\prime}$ denotes that the sum includes faces $f_{i, x}$ on the fine lattice that belong to the face $f_{i, x^{\prime}}^{\prime}$ on the coarse lattice. Of course, by construction the coarse lattice velocity variables also obey the continuity equation. This block factor 2 renormalization 
group transformation (RGT) will be used later to demonstrate that the structure of the perfect equations of motion reproduces itself under renormalization.

\section{Perfect equations of motion for Hagen-Poiseuille flow}

For simplicity we first discuss the derivation of perfect equations of motion in the context of Hagen-Poiseuille flow. The general equations for static, slow flows are derived in section 6 . We start from the Navier-Stokes equation

$$
\partial_{t} v_{i}(y)+\left(v_{j}(y) \partial_{j}\right) v_{i}(y)=-\frac{1}{\rho} \partial_{i} p(y)+\nu \partial_{j} \partial_{j} v_{i}(y)
$$

Here $\nu$ is the kinematic viscosity of the fluid, and $\rho$ is its density. We restrict ourselves to incompressible flows, i.e. to a constant density, and thus the continuity equation applies. The appropriate boundary condition for a viscous fluid at a fixed wall is $v_{i}(y)=0$ for all $i$. Furthermore, we consider static Hagen-Poiseuille flow, i.e. a constant pressure gradient $\delta p$, in a channel with square cross section $A=L^{d-1}$ along the $d$-direction. Hence, the pressure is given by $p(y)=\delta p y_{d}$. The velocity is along the channel, i.e. only $v_{d}(y)$ is nonzero and in addition independent of $y_{d}$. Then the equations reduce to the $(d-1)$-dimensional Poisson equation

$$
\partial_{i} \partial_{i} v_{d}(y)=\frac{\delta p}{\nu \rho}
$$

with Dirichlet boundary conditions $v_{d}(y)=0$ at the edge of the square. This equation follows from a variational principle of an action

$$
S\left[v_{d}\right]=\int_{A} d^{d-1} y\left[\frac{1}{2} \partial_{i} v_{d}(y) \partial_{i} v_{d}(y)+\frac{\delta p}{\nu \rho} v_{d}(y)\right] .
$$

Note that here $i$ runs from 1 to $(d-1)$ only. The action is useful in our derivation of the perfect equations of motion. Yet we want to stress that the existence of an action is not really necessary. This is crucial because there is no action principle for the full Navier-Stokes problem.

What is the perfect discretization of the Poisson equation? To answer that question we consider the large scale lattice variable

$$
V_{d, x}=\frac{1}{a^{d-1}} \int_{f_{d, x}} d^{d-1} y v_{d}(y)
$$

Note that the face $f_{d, x}$ is nothing more than a $(d-1)$-dimensional cube, and $V_{d, x}$ behaves practically as a $(d-1)$-dimensional scalar. We want to impose eq.(3.4) as 
a constraint for each face $f_{d, x}$. For this purpose we add it to the action using a Lagrange multiplier field $\eta_{d, x}$ that lives on the face $f_{d, x}$

$$
\begin{aligned}
S\left[v_{d}, V_{d}, \eta_{d}\right] & =\int_{A} d^{d-1} y\left[\frac{1}{2} \partial_{i} v_{d}(y) \partial_{i} v_{d}(y)+\frac{\delta p}{\nu \rho} v_{d}(y)\right] \\
& +\sum_{x} a^{d-1}\left[\eta_{d, x}\left(V_{d, x}-\frac{1}{a^{d-1}} \int_{f_{d, x}} d^{d-1} y v_{d}(y)\right)-\frac{a^{2} \alpha}{2} \eta_{d, x}^{2}\right]
\end{aligned}
$$

We have also included a term quadratic in the Lagrange multiplier field. This would not really be necessary, but its coefficient $\alpha$ will allow us to optimize the perfect equation's locality, and therefore it is of great practical importance. Next we derive equations of motion from the variational principle of the action. Varying the action with respect to $v_{d}(y)$ yields

$$
\partial_{i} \partial_{i} v_{d}(y)-\frac{\delta p}{\nu \rho}+\sum_{x} \eta_{d, x} \theta_{d, x}(y)=0,
$$

where $\theta_{d, x}(y)=1$ for $y \in f_{d, x}$ and zero otherwise. Varying the action with respect to $\eta_{d, x}$ one obtains

$$
V_{d, x}-\frac{1}{a^{d-1}} \int_{f_{d, x}} d^{d-1} y v_{d}(y)-a^{2} \alpha \eta_{d, x}=0 .
$$

It appears that the constraint of eq.(3.4) is correctly implemented only for $\alpha=0$. However, varying the action with respect to $V_{d, x}$ gives

$$
\eta_{d, x}=0
$$

To solve the above equations we now go to momentum space. Since the velocity vanishes at the boundary the appropriate Fourier transform takes the form

$$
v_{d}(k)=\int_{A} d^{d-1} y v_{d}(y) \prod_{i=1}^{d-1} \sin \left(k_{i} y_{i}\right) .
$$

Here the

$$
k_{i}=\frac{\pi}{L} m_{i}
$$

are discrete momenta with $m_{i} \in \mathbf{N}$. Note that $v_{d}(k)$ extends naturally to negative momentum values because

$$
v_{d}\left(k_{1}, \ldots,-k_{i}, \ldots, k_{d-1}\right)=-v_{d}\left(k_{1}, \ldots, k_{i}, \ldots, k_{d-1}\right) .
$$

Similarly, for the lattice velocity field we have

$$
V_{d}(k)=a^{d-1} \sum_{x} V_{d, x} \prod_{i=1}^{d-1} \sin \left(k_{i} y_{i}\right) .
$$


Now the sum is finite and extends over $m_{i}=1,2, \ldots, N$ only, where $N=L / a$. The Lagrange multiplier field $\eta_{d, x}$ is transformed analogously. In Fourier space the action takes the form

$$
\begin{aligned}
& S\left[v_{d}, V_{d}, \eta_{d}\right]=\frac{1}{a^{d-1}} \sum_{k \in\left(\frac{\pi N}{L}\right)^{d-1}}\left[\frac{1}{2} k^{2} v_{d}(k)^{2}+\frac{\delta p}{\nu \rho} \delta_{L}(k) v_{d}(k)\right] \\
& +\frac{1}{a^{d-1}} \sum_{k}\left[\left(V_{d}(k)-\sum_{l \in \mathbf{Z}^{d-1}} v_{d}(k+2 \pi l / a) \Pi_{d}(k+2 \pi l / a) \prod_{i=1}^{d-1}(-1)^{l_{i}}\right) \eta_{d}(k)\right. \\
& \left.-\quad \frac{a^{2} \alpha}{2} \eta_{d}(k)^{2}\right] .
\end{aligned}
$$

The second sum over $k$ is restricted to $m_{i} \in\{1,2, \ldots, N\}$. In the above expression we have introduced

$$
\delta_{L}(k)=\prod_{i=1}^{d-1} \frac{1-(-1)^{m_{i}}}{k_{i}}
$$

as the Fourier transform of the constant 1. The function

$$
\Pi_{d}(k)=\prod_{i=1}^{d-1} \frac{2 \sin \left(k_{i} a / 2\right)}{k_{i} a}
$$

results from the Fourier transform of $\theta_{d, x}(y)$. In Fourier space eq.(3.6) takes the form

$$
-k^{2} v_{d}(k)-\frac{\delta p}{\nu \rho} \delta_{L}(k)+\Pi_{d}(k) \eta_{d}(k)=0
$$

In eq.(3.16) the momentum $k$ has the form of eq.(3.10) with $m_{i} \in N$ being an arbitrary integer. On the other hand, as it stands $\eta_{d}(k)$ is defined only for momenta with $m_{i} \leq N$. For other momenta $\eta_{d}(k)$ naturally extends to

$$
\begin{aligned}
& \eta_{d}\left(k_{1}, \ldots,-k_{i}, \ldots, k_{d-1}\right)=-\eta_{d}\left(k_{1}, \ldots, k_{i}, \ldots, k_{d-1}\right), \\
& \eta_{d}\left(k+2 \pi l_{i} \hat{i} / a\right)=(-1)^{l_{i}} \eta_{d}(k) .
\end{aligned}
$$

Solving eq.(3.16) yields

$$
v_{d}(k)=\frac{1}{k^{2}}\left[\Pi_{d}(k) \eta_{d}(k)-\frac{\delta p}{\nu \rho} \delta_{L}(k)\right] .
$$

We plug this back into the action and obtain

$$
S\left[V_{d}, \eta_{d}\right]=\frac{1}{a^{d-1}} \sum_{k}\left[-\frac{1}{2} \eta_{d}(k) \omega_{d d}(k)^{-1} \eta_{d}(k)+\frac{\delta p}{\nu \rho} \Delta_{L}(k) \eta_{d}(k)+V_{d}(k) \eta_{d}(k)\right],
$$

where

$$
\begin{aligned}
\omega_{d d}(k)^{-1} & =\sum_{l \in \mathbf{Z}^{d-1}} \frac{1}{(k+2 \pi l / a)^{2}} \Pi(k+2 \pi l / a)^{2}+\alpha a^{2} \\
\Delta_{L}(k) & =\sum_{l \in \mathbf{Z}^{d-1}} \delta_{L}(k+2 \pi l / a) \frac{1}{(k+2 \pi l / a)^{2}} \Pi_{d}(k+2 \pi l / a)(-1)^{\sum_{i} l_{i}} .
\end{aligned}
$$


Minimizing with respect to the auxiliary field $\eta_{d}(k)$ yields

$$
\eta_{d}(k)=\omega_{d d}(k)\left(V_{d}(k)+\frac{\delta p}{\nu \rho} \Delta_{L}(k)\right) .
$$

Reinserting this into the action finally gives

$$
S\left[V_{d}\right]=\frac{1}{a^{d-1}} \sum_{k}\left[\frac{1}{2} V_{d}(k) \omega_{d d}(k) V_{d}(k)+\omega_{d d}(k) \frac{\delta p}{\nu \rho} \Delta_{L}(k) V_{d}(k)\right] .
$$

This is a perfect action for the Poisson equation in a finite volume. The resulting perfect equation of motion takes the form

$$
\omega_{d d}(k) V_{d}(k)+\omega_{d d}(k) \frac{\delta p}{\nu \rho} \Delta_{L}(k)=0 .
$$

By construction it is clear that this equation perfectly represents continuum physics. It was helpful to have an action whose variation produced the perfect equation of motion, but the action was not really necessary. As we will see later, all we really need are the equations of motion. This is important for the full Navier-Stokes problem, for which an action does not exist.

For practical applications eq.(3.23) needs to be transformed back to coordinate space. It should be noted that $\omega_{d d}(k)$ contains the arbitrary parameter $\alpha$. Hence, eq. (3.23) represents a whole family of perfect equations of motion. Now we want to optimize $\alpha$ such that the equations become as local as possible. For this purpose we consider $d=2$. Then the sums in eq.(3.20) can be performed analytically, and one obtains

$$
\begin{aligned}
\omega_{22}\left(k_{1}\right)^{-1} & =a^{2}\left[\frac{1}{4 \sin ^{2}\left(a k_{1} / 2\right)}-\frac{1}{6}+\alpha\right] \\
\Delta_{L}\left(k_{1}\right) & =a^{3}\left[\frac{1}{8 \sin ^{3}\left(a k_{1} / 2\right)}-\frac{1}{6} \frac{1}{2 \sin \left(a k_{1} / 2\right)}\right]\left(1-(-1)^{m_{1}}\right),
\end{aligned}
$$

where again $k_{1}=\pi m_{1} / L$.

For the choice of $\alpha=1 / 6$ this gives $\omega_{22}\left(k_{1}\right)=4 / a^{2} \sin ^{2}\left(a k_{1} / 2\right)$, which corresponds to the standard ultralocal second derivative in coordinate space. Transforming the whole perfect equation of motion eq.(3.23) to coordinate space one obtains

$$
\frac{1}{a^{2}}\left[V_{2, x_{1}+a}-2 V_{2, x_{1}}+V_{2, x_{1}-a}\right]=\frac{\delta p}{\nu \rho},
$$

for $x_{1}=(n-1 / 2) a$ with $n \neq 1, N$, i.e. for points away from the boundary. At the left boundary one finds

$$
\frac{1}{a^{2}}\left[V_{2,3 a / 2}-3 V_{2, a / 2}\right]=\frac{2}{3} \frac{\delta p}{\nu \rho}
$$




\begin{tabular}{|c|c|c|c|c|c|}
\hline$V_{(1 / 2,1 / 2)}$ & $x=1 / 2$ & $x=3 / 2$ & $x=5 / 2$ & $x=7 / 2$ & $x=9 / 2$ \\
\hline$y=1 / 2$ & 4.28599 & -0.42637 & -0.00118 & -0.00013 & -0.00000 \\
\hline$y=3 / 2$ & -0.42637 & -0.18734 & -0.00227 & -0.00003 & -0.00000 \\
\hline$y=5 / 2$ & -0.00118 & -0.00227 & 0.00151 & 0.00006 & 0.00000 \\
\hline$y=7 / 2$ & -0.00013 & -0.00003 & 0.00006 & -0.00001 & 0.00000 \\
\hline$y=9 / 2$ & -0.00000 & -0.00000 & 0.00000 & 0.00000 & 0.00000 \\
\hline
\end{tabular}

Table 1: Coupling of a corner field

\begin{tabular}{|c|c|c|c|c|c|}
\hline$V_{(5 / 2,5 / 2)}$ & $x=1 / 2$ & $x=3 / 2$ & $x=5 / 2$ & $x=7 / 2$ & $x=9 / 2$ \\
\hline$y=1 / 2$ & 0.00151 & -0.00070 & -0.00188 & -0.00070 & 0.00151 \\
\hline$y=3 / 2$ & -00070 & -0.19033 & -0.61801 & -0.19033 & -0.00070 \\
\hline$y=5 / 2$ & -0.00188 & -0.61801 & 3.24027 & -0.61801 & -0.00188 \\
\hline$y=7 / 2$ & -0.00070 & -0.19033 & -0.61801 & -0.19033 & -0.00070 \\
\hline$y=9 / 2$ & 0.00151 & -0.00070 & -0.00188 & -0.00070 & 0.00151 \\
\hline
\end{tabular}

Table 2: Coupling of a center field

and there is an analogous expression at the right boundary. It is straightforward to verify that the averaged continuum solution does in fact satisfy these equations. In the continuum the Hagen-Poiseuille flow in $d=2$ is given by

$$
v_{2}\left(y_{1}\right)=\frac{1}{2} \frac{\delta p}{\nu \rho} y_{1}\left(y_{1}-L\right)
$$

Consequently, the corresponding large scale lattice variable takes the form

$$
V_{2, x_{1}}=\frac{1}{a} \int_{x_{1}-a / 2}^{x_{1}+a / 2} d y_{1} v_{2}\left(y_{1}\right)=\frac{1}{2} \frac{\delta p}{\nu \rho}\left[x_{1}\left(x_{1}-L\right)+\frac{a^{2}}{12}\right]
$$

which indeed solves eq.(3.25).

In $d=3$ the perfect equations of motion are no longer ultralocal. Still, when we use the optimized $\alpha=1 / 6$ from $d=2$ also in 3 dimensions the equations turn out to be extremely local. In coordinate space the equation of motion takes the form

$$
\sum_{x^{\prime}} \omega_{d d}\left(x, x^{\prime}\right) V_{d, x^{\prime}}=-\frac{\delta p}{\nu \rho} \Delta_{L}(x)
$$

where $\omega_{d d}\left(x, x^{\prime}\right)$ and $\Delta_{L}(x)$ are Fourier transforms of the corresponding quantities in momentum space. Their values for a 3-d system with $N=5, a=1$ are given in tables 1, 2 and 3 for various values of $x$ and plots for the same values are shown in figures 2, 3, and 4. Also the values for $D_{L}(x)$ (with $\delta p / \nu \rho$ set to 1 ) are provided in table 4, and displayed in Fig. 5. 


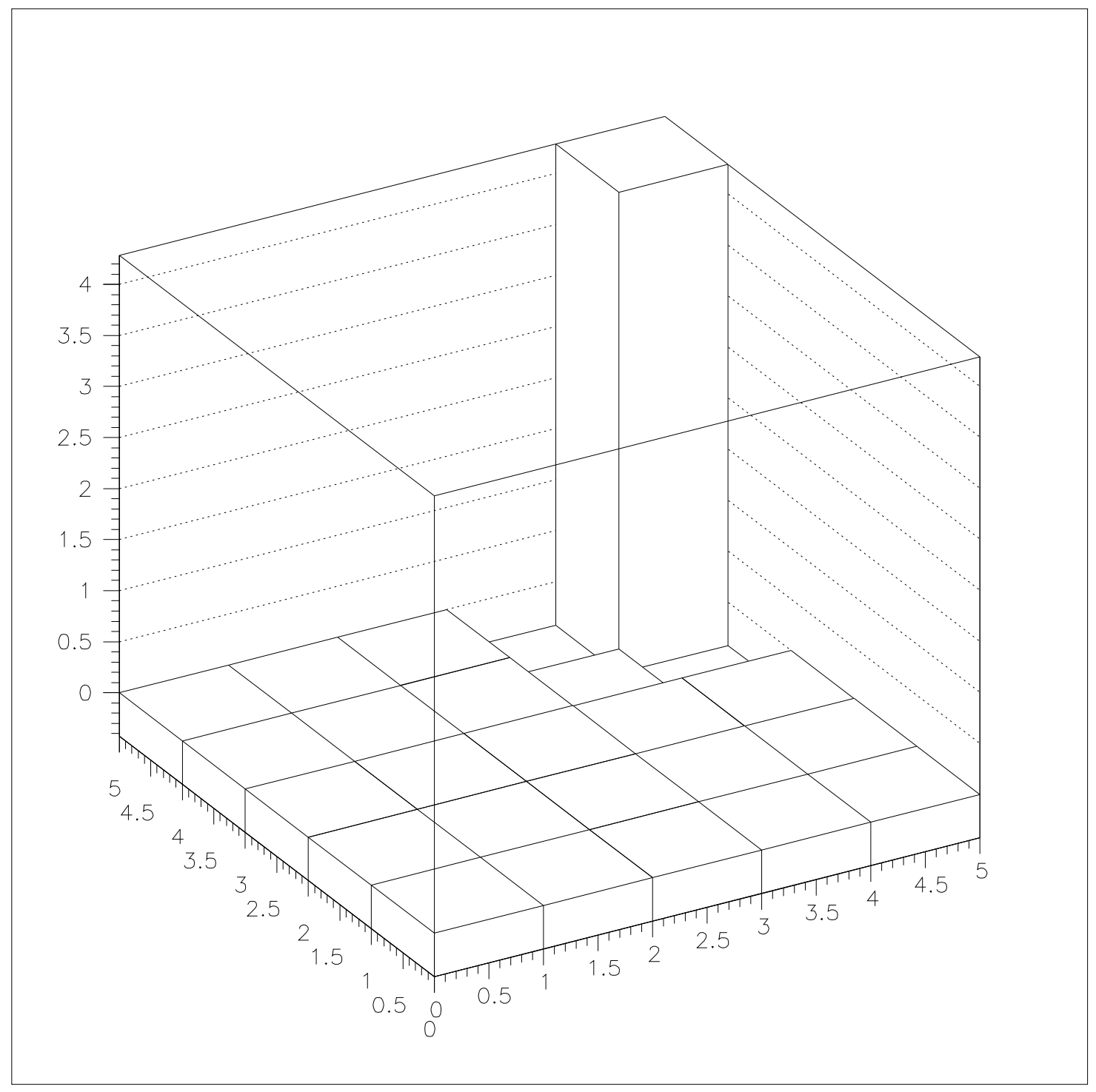

Figure 2: The coupling of a corner field to the lattice

\section{Invariance of the lattice equations of motion un- der a factor 2 RGT}

We would like to show explicitly that the lattice equations of motions remain unchanged under a factor 2 renormalization group transformation. To do this we must first find the recursion relation, relating the equations on the coarse lattice to the ones on the fine lattice. To find the recursion relation, we add to the action in the fine lattice variables $V_{d, x}$ (with spacing $a$ ) the constraint involving the coarse lattice 


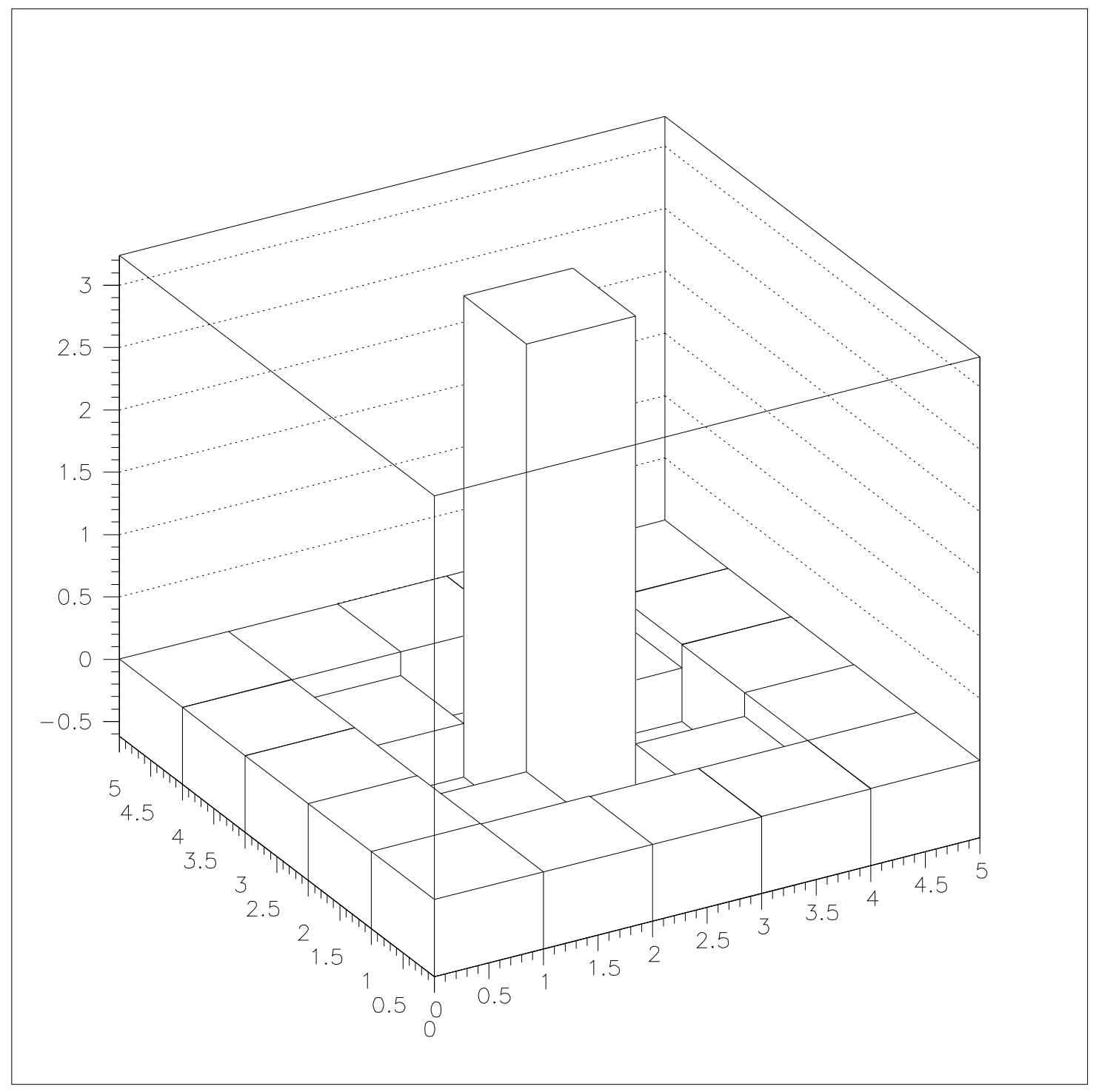

Figure 3: The coupling of a center field to the lattice

variables $V_{d, x^{\prime}}^{\prime}($ with spacing $2 a)$,

$$
V_{d, x^{\prime}}^{\prime}=\frac{1}{2^{d-1}} \sum_{x \in x^{\prime}} V_{d, x},
$$

using a Lagrange multiplier $\eta_{d, x^{\prime}}$. Varying the action with respect to the various fields yields equations analagous to the ones relating the continuum fields to the lattice fields

$$
\begin{gathered}
\sum_{y} V_{y} \omega_{d d}(y, x)+\frac{\delta p}{\nu \rho} D_{L}(x)-\frac{1}{2^{d-1}} \sum_{x} \theta_{d, x^{\prime}} \eta_{d, x^{\prime}}(x)=0 \\
V_{d, x^{\prime}}^{\prime}-\frac{1}{2^{d-1}} \sum_{x \in x^{\prime}} V_{d, x}-\alpha_{2} \eta_{d, x^{\prime}}=0
\end{gathered}
$$




\begin{tabular}{|c|c|c|c|c|c|}
\hline$V_{(5 / 2,1 / 2)}$ & $x=1 / 2$ & $x=3 / 2$ & $x=5 / 2$ & $x=7 / 2$ & $x=9 / 2$ \\
\hline$y=1 / 2$ & -0.00118 & -0.42769 & 3.85830 & -0.42769 & -0.00118 \\
\hline$y=3 / 2$ & -0.00227 & -0.18965 & -0.61602 & -0.18965 & -0.00227 \\
\hline$y=5 / 2$ & -0.00151 & -0.00070 & -0.00188 & -0.00070 & -0.00151 \\
\hline$y=7 / 2$ & 0.00006 & 0.00002 & -0.00011 & 0.00002 & 0.00006 \\
\hline$y=9 / 2$ & 0.00000 & 0.00000 & -0.00000 & 0.00000 & 0.00000 \\
\hline
\end{tabular}

Table 3: Coupling of an edge field

\begin{tabular}{|c|c|c|c|c|c|}
\hline$*$ & $x=1 / 2$ & $x=3 / 2$ & $x=5 / 2$ & $x=7 / 2$ & $x=9 / 2$ \\
\hline$y=1 / 2$ & 0.45985 & 0.66567 & 0.66624 & 0.66567 & 0.45985 \\
\hline$y=3 / 2$ & 0.66567 & 0.99806 & 0.99902 & 0.99806 & 0.66567 \\
\hline$y=5 / 2$ & 0.66624 & 0.99902 & 0.99894 & 0.99902 & 0.66624 \\
\hline$y=7 / 2$ & 0.66567 & 0.99806 & 0.99902 & 0.99806 & 0.66567 \\
\hline$y=9 / 2$ & 0.45985 & 0.66567 & 0.66624 & 0.66567 & 0.45985 \\
\hline
\end{tabular}

Table 4: The values of $D_{L}(x)$ for $\delta p / \nu \rho$ set to 1

$$
\eta_{d, x^{\prime}}=0
$$

Here $\theta_{d, x^{\prime}}(x)=1$ for $x \in x^{\prime}$ and zero otherwise and $\alpha_{2}$ is a constant to be adjusted to fit with our earlier choice of optimization parameter $\alpha$. Fourier transforming the above equations gives

$$
\begin{aligned}
& V_{d}(k) \omega_{d d}(k)+\frac{\delta p}{\nu \rho} \omega_{d d}(k) \Delta_{L}(k)-\tilde{\Pi}(k) \eta_{d}(k)=0 \\
& V_{d}^{\prime}\left(k^{\prime}\right)-\sum_{m \in\{0,1\}^{d-1}} V_{d}\left(k^{\prime}+\pi m / a\right) \tilde{\Pi}\left(k^{\prime}+\pi m / a\right) \prod_{i=1}^{d-1}(-1)_{i}^{l}-\alpha_{2} \eta\left(k^{\prime}\right)=0 \\
\eta\left(k^{\prime}\right)= & 0
\end{aligned}
$$

where $k_{i}^{\prime}=\pi / L n_{i}$ with $n_{i} \in\{1, N / 2\}$ only, and

$$
\tilde{\Pi}(k)=\prod_{i=1}^{d-1} \cos \left(k_{i} a / 2\right)=\frac{\Pi(2 k)}{\Pi(k)}
$$

is the Fourier transform of $\theta_{x}^{\prime}(x)$. We note that in the above equations $\eta_{d}\left(k^{\prime}\right)$ naturally extends to momenta $n_{i}>N / 2$ by

$$
\eta_{d}\left(k^{\prime}+\pi m_{i} \hat{i} / a\right)=(-1)^{m_{i}} \eta_{d}\left(k^{\prime}\right) .
$$

Solving the first equation for $V_{d}(k)$ we obtain

$$
V_{d}(k)=\frac{1}{2^{d-1}} \omega(k)^{-1} \tilde{\Pi}(k)-\frac{\delta p}{\nu \rho} \Delta_{L}(k) .
$$




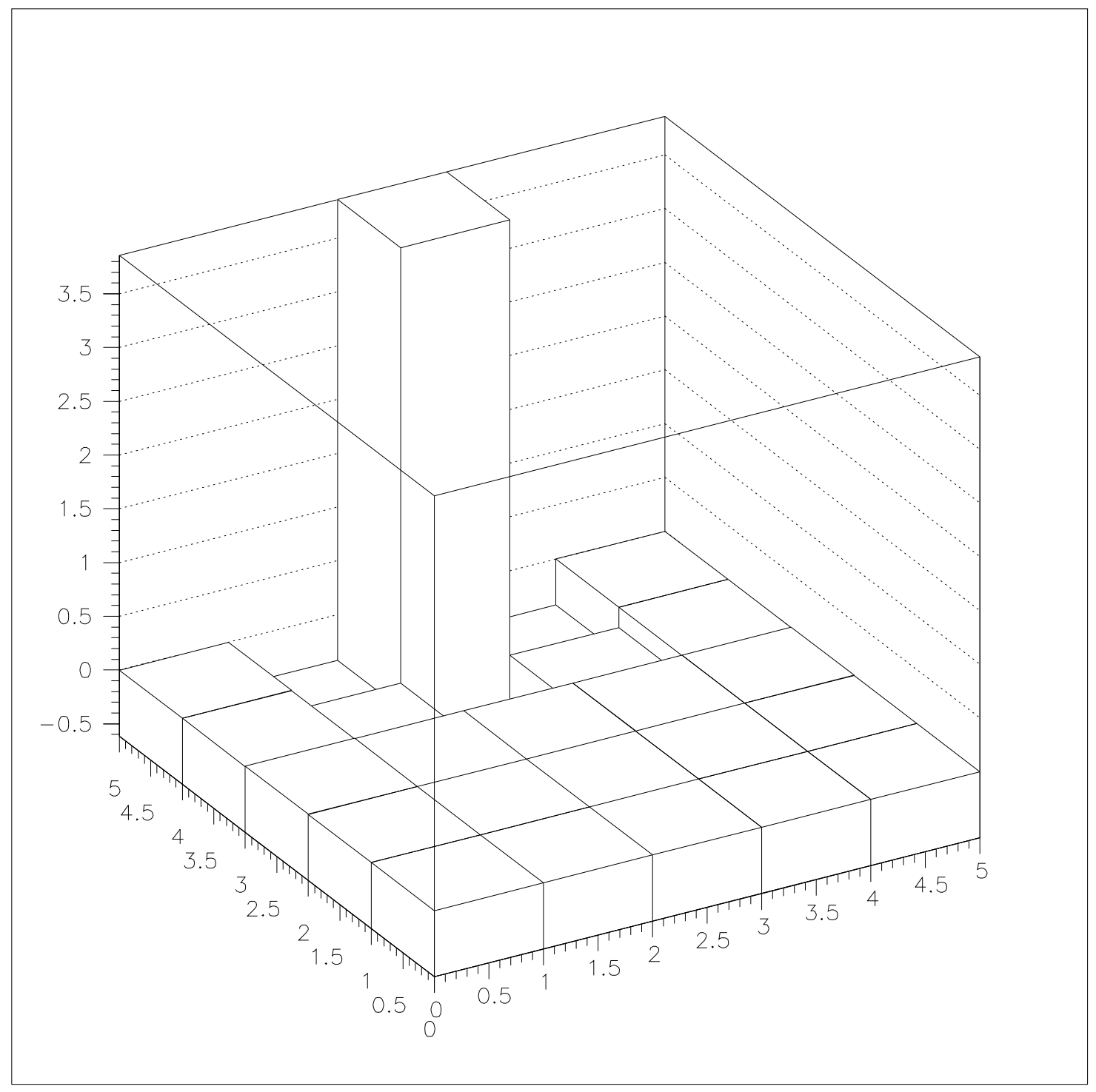

Figure 4: The coupling of an edge field to the lattice

Inserting this relation into the other two equations and comparing with eq.(3.23) yields recursion formulas for $\omega_{d d}(k)$ and $\Delta_{L}(k)$ :

$$
\begin{aligned}
\omega_{d d}^{\prime}\left(k^{\prime}\right)^{-1} & =\sum_{m \in\{0,1\}^{d-1}} \omega_{d d}\left(k^{\prime}+\pi m / a\right)^{-1} \tilde{\Pi}\left(k^{\prime}+\pi m / a\right)^{2}+\alpha_{2} \\
\Delta_{L}^{\prime}\left(k^{\prime}\right) & =\sum_{m \in\{0,1\}^{d-1}} \Delta_{L}\left(k^{\prime}+\pi m / a\right) \tilde{\Pi}\left(k^{\prime}+\pi m / a\right)(-1)^{\sum_{i} m_{i}} .
\end{aligned}
$$

We now plug in the explicit form of $\omega_{d d}(k)$ from eq.(3.20) into the first recursion relation

$$
\omega_{d d}^{\prime}\left(k^{\prime}\right)^{-1}=\sum_{m \in\{0,1\}^{d-1}} \sum_{l \in \mathbf{Z}^{d-1}} \frac{1}{\left(k^{\prime}+\pi(2 l+m) / a\right)^{2}} \Pi\left(2 k^{\prime}+2 \pi(2 l+m) / a\right)^{2}
$$




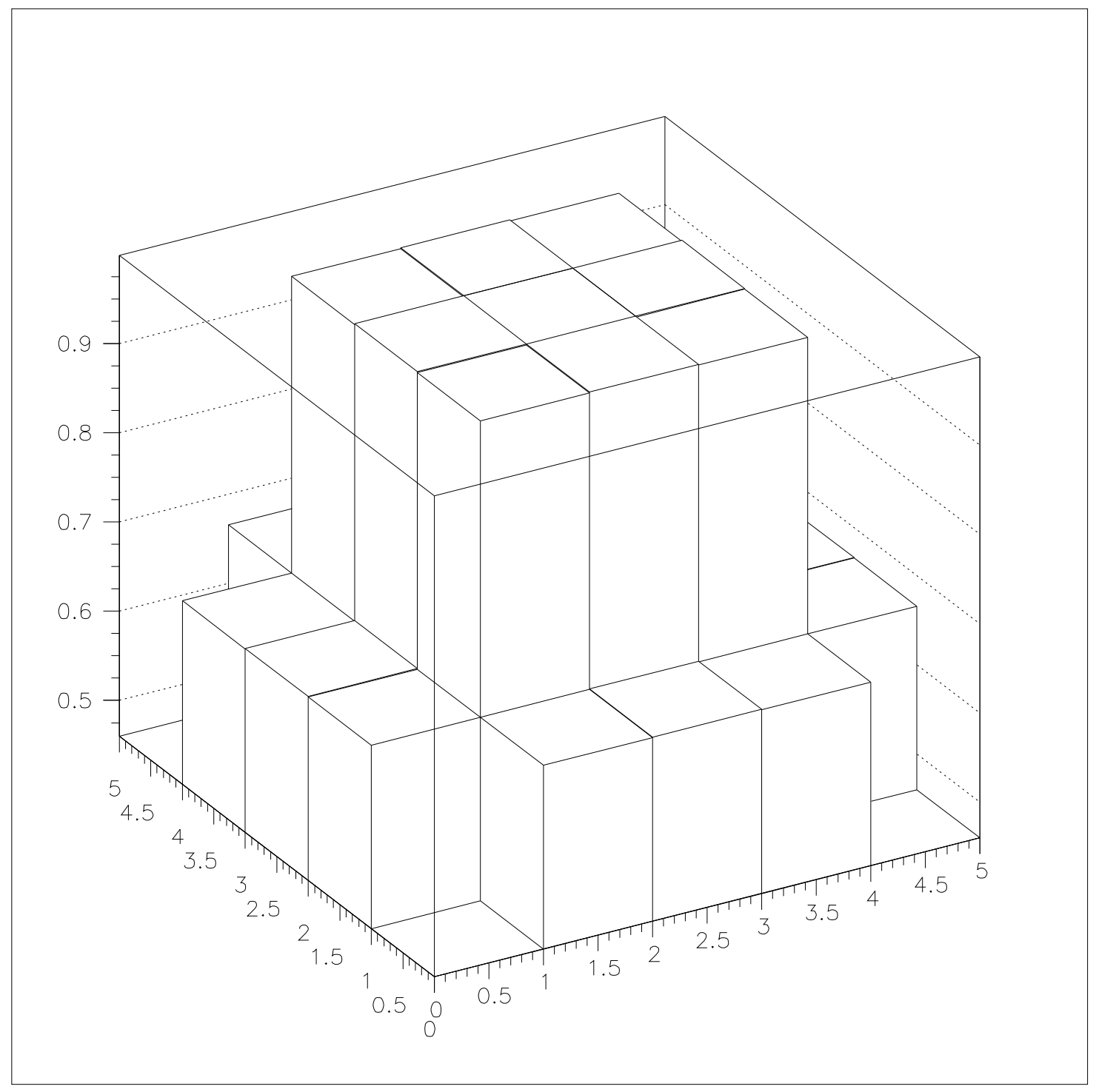

Figure 5: Values of $D_{L}(x)$

$$
\begin{aligned}
& +a^{2} \alpha \frac{\Pi\left(2\left(k^{\prime}+\pi m\right)\right)^{2}}{\Pi\left(k^{\prime}+\pi m\right)^{2}}+\alpha_{2} \\
& =\sum_{l^{\prime} \in \mathbf{Z}^{d-1}} \frac{1}{\left(k^{\prime}+2 \pi l^{\prime} / a^{\prime}\right)^{2}} \prod_{i=1}^{d-1} \frac{4 \sin ^{2}\left(a^{\prime} k_{i}^{\prime}+2 \pi l_{i}^{\prime}\right)}{a^{\prime} k_{i}^{\prime}+2 \pi l_{i}^{\prime}}+a^{2} \alpha+\alpha_{2},
\end{aligned}
$$

where we have let $l_{i}^{\prime}=2 l_{i}+m_{i}$ and $a^{\prime}=2 a$ in the last step. Now, letting $\alpha_{2}=3 a^{2} \alpha$ we recover the familiar form for $\omega_{d d}^{\prime}\left(k^{\prime}\right)$. Thus, we see that under the factor 2 recursion relation, $\omega_{d d}(k)$ for lattice spacing $a$ transforms into $\omega_{d d}^{\prime}\left(k^{\prime}\right)$ for lattice spacing $2 a$. It can be shown that $\Delta_{L}(k)$ behaves similarly. The fact that the equations transform properly indeed confirms the they are perfect lattice equations of motion.

The recursion relation also serves as a powerful tool for numerical methods be- 


\begin{tabular}{|c|c|c|c|}
\hline$*$ & $x=1 / 2$ & $x=3 / 2$ & $x=5 / 2$ \\
\hline$y=1 / 2$ & 0.81972 & 1.40674 & 0.81972 \\
\hline$y=3 / 2$ & 1.40674 & 2.48867 & 1.40674 \\
\hline$y=5 / 2$ & 0.81972 & 1.40674 & 0.81972 \\
\hline
\end{tabular}

Table 5: Velocity field values for $N=3$

\begin{tabular}{|c|c|c|c|c|c|c|}
\hline$*$ & $x=1 / 2$ & $x=3 / 2$ & $x=5 / 2$ & $x=7 / 2$ & $x=9 / 2$ & $x=11 / 2$ \\
\hline$y=1 / 2$ & 0.31513 & 0.68669 & 0.83510 & 0.83510 & 0.68669 & 0.31513 \\
\hline$y=3 / 2$ & 0.68669 & 1.58922 & 1.97725 & 1.97725 & 1.58992 & 0.68669 \\
\hline$y=5 / 2$ & 0.83510 & 1.97725 & 2.48758 & 2.48758 & 1.97725 & 0.83510 \\
\hline$y=7 / 2$ & 0.83510 & 1.97725 & 2.48758 & 2.48758 & 1.97725 & 0.83510 \\
\hline$y=9 / 2$ & 0.68669 & 1.58922 & 1.97725 & 1.97725 & 1.58992 & 0.68669 \\
\hline$y=11 / 2$ & 0.31513 & 0.68669 & 0.83510 & 0.83510 & 0.68669 & 0.31513 \\
\hline
\end{tabular}

Table 6: Velocity field for $N=6$

cause of the locality of the couplings. For example, one cannot solve analytically the lattice equations for a rectangular cross section from which a smaller rectangular slice has been removed. However, eq.(4.2) is not specific to a given channel geometry and hence it still applies. To find the coupings for inward corner points one need only choose a very fine lattice. On it specify the couplings already known, make ultralocal guesses for the unknown sites, and perform several recursions. Since the couplings are so local, if the lattice is fine enough they serve as a very good approximation to the actual values that define the equations of motion. Thus, the averaging process of the recursive step will tend to converge to fixed values, therby providing the necessary information for the unkown sites. In fact, we could have derived all the couplings in the above tables in this fashion by making ultralocal naive guesses for the Poissonian and then performing recursions.

In addition, we have numerically calculated the averaged velocity field for $N=6$, $a=1$ and for $N=3, a=2$. This was done by first numerically transforming $\omega_{d d}(k)$ and $\omega_{d d}(k) \Delta_{L}(k)$ back into coordinate space. The values obtained were then used to solve eq.(3.29) for the lattice velocity field. The solution was found iteratively by making an initial guess for the field values and then using the equation of motion to find the field at $x$ in terms of the fields at $x^{\prime} \neq x$. With each iterative step the velocity field at $x$ was replaced (if different) by the value of the field as found through the equation of motion. The iteration was stopped once the field converged to stationary values. For the above cases the field values are provided in tables 5, 6 and plotted in figures 6 and 7 . We have explcitly checked that a factor 2 RGT on the fine solution gives the coarse lattice fields values. The fact that they satisfy eq.(3.4) confirms that the equations of motion found are perfect. 


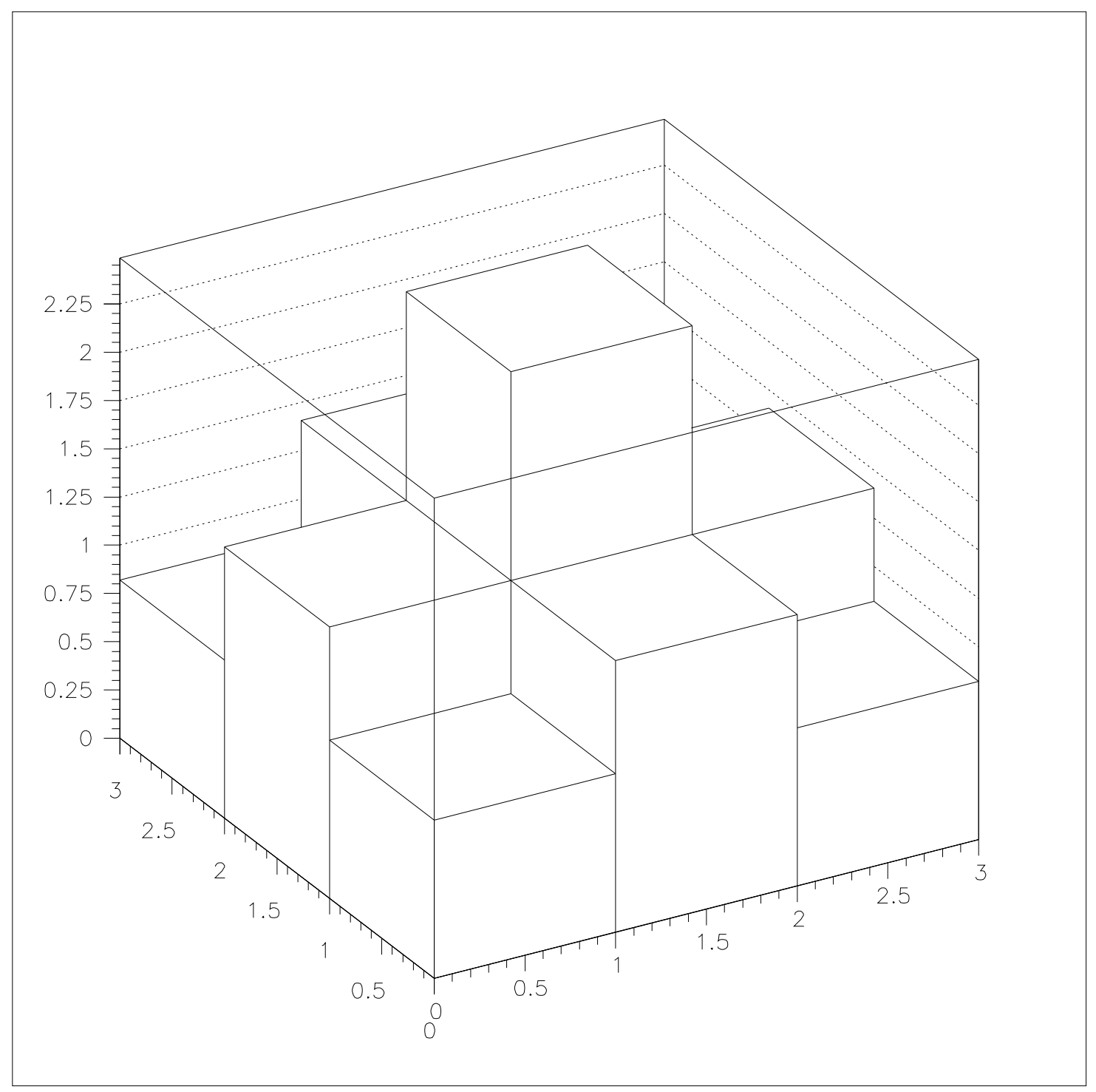

Figure 6: The velocity field for $N=3$

\section{The perfect velocity field}

Up to now we have shown that the perfect lattice equations of motion give exact results for the continuum fields averaged over cubes. In many practical cases one is most interested in these averaged quantities. Still, in some cases one would also like to know the values of the continuum fields themselves. In particular, when one works on a very coarse lattice the averaged lattice quantities may not contain enough information, and one would like to extract information for continuum points. Fortunately, this is possible with a perfect discretization. In fact, one can reconstruct the continuum field from the lattice data. For this purpose we insert eq.(3.21) into 


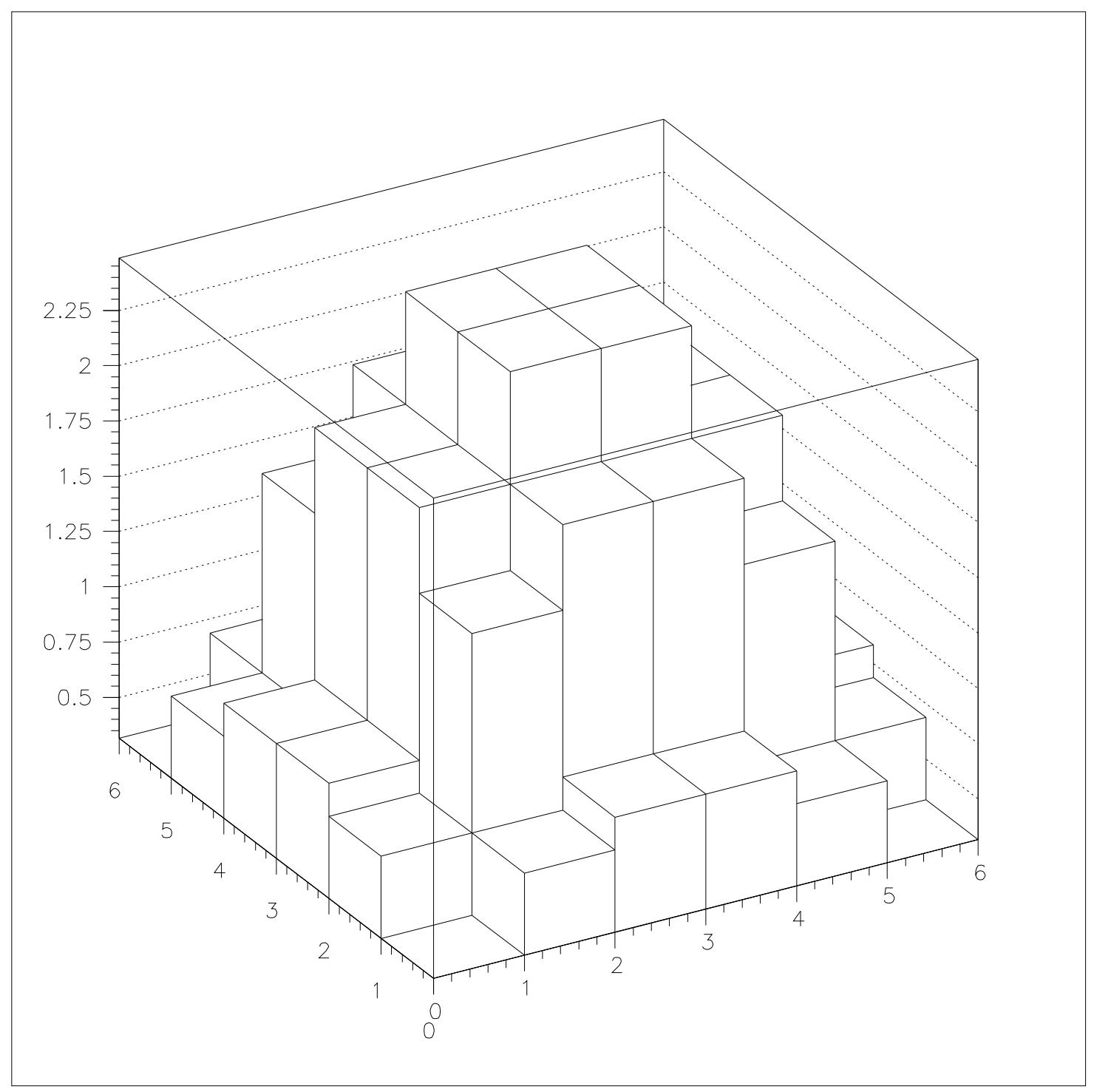

Figure 7: The velocity field for $N=6$

eq.(3.18) and obtain

$$
v_{d}(k)=\frac{1}{k^{2}}\left[\Pi_{d}(k) \omega_{d d}(k)\left(\frac{1}{a^{2}} V_{d}(k)+\frac{\delta p}{\nu \rho} \Delta_{L}(k)\right)-\frac{\delta p}{\nu \rho} \delta_{L}(k)\right],
$$

which gives the continuum velocity field in terms of the lattice field $V_{d}$. Transforming back to coordinate space one can reconstruct exactly the continuum velocity field. When one considers the continuum velocity field at lattice points one finds

$$
v_{d}(x)=\sum_{x^{\prime}} \rho_{d d}\left(x, x^{\prime}\right) V_{d, x^{\prime}}+\frac{\delta p}{\nu \rho} \lambda_{d}(x) .
$$


For a 2-d system $\rho_{d d}$ and $\lambda_{d}$ are ultralocal and

$$
v_{d}(x)=\frac{1}{8}\left(V_{d, x+a}+6 V_{d, x}+V_{d, x-a}\right)-\frac{1}{3} \frac{\delta p}{\nu \rho}
$$

for $x \neq a / 2,(N-1 / 2) a$ and for the boundary point $x=a / 2$

$$
v_{d}(a / 2)=\frac{1}{8}\left(5 V_{d, a / 2}+V_{d, 3 a / 2}\right)-\frac{1}{3} \frac{\delta p}{\nu \rho} .
$$

For continuum points which are not lattice points one can also obtain the values of $\rho_{d d}$ and $\lambda_{d}$ by Fourier transformation.

\section{Perfect discretization for static slow flows}

Now we turn to general static flows with not necessarily constant pressure gradients. In this case the Navier-Stokes equation reduces to

$$
\partial_{j} \partial_{j} v_{i}(y)=\frac{1}{\nu \rho} \partial_{i} p(y)
$$

For reasons of simplicity we restrict ourselves to an infinite volume. The inclusion of boundary conditions is straightforward (though tedious) and can be done in analogy to the Hagen-Poiseuille flow. Although the above equation still follows from an action principle, here we restrict ourselves to working with the equations of motion alone. Eq.(3.6) then takes the form

$$
\partial_{i} \partial_{i} v_{j}(y)-\frac{1}{\nu \rho} \partial_{j} p+\sum_{x} \eta_{j, x} \theta_{j, x}(y)=0
$$

and we still have

$$
V_{j, x}-\frac{1}{a^{d-1}} \int_{f_{j, x}} d^{d-1} y v_{j}(y)-a^{2} \alpha \eta_{j, x}=0, \eta_{j, x}=0 .
$$

Similarly, the continuity equation leads to

$$
\frac{1}{\nu \rho} \partial_{i} v_{i}(y)+\sum_{x} \eta_{x} \theta_{x}(y)=0
$$

and we also have

$$
P_{x}-\frac{1}{a^{d}} \int_{c_{x}} d^{d} y p(y)-a^{2} \beta \eta_{x}=0, \eta_{x}=0 .
$$

Note that we have included a parameter $\beta$ analagous to $\alpha$ to be optimized for locality later. It will turn out that this is not necessary, because the equations of motion 
can be optimized for locality without it. Since we are now in an infinite volume

$$
\begin{aligned}
v_{j}(k) & =\int d^{d} y v_{j}(y) \prod_{i=1}^{d} \exp \left(-i k_{i} y_{i}\right), \\
p(k) & =\int d^{d} y p(y) \prod_{i=1}^{d} \exp \left(-i k_{i} y_{i}\right),
\end{aligned}
$$

where $k \in \mathbf{R}^{d}$. Hence, it is conveniant to now let

$$
\begin{aligned}
V_{j}(k) & =a^{d-1} \sum_{x} V_{j, x} \prod_{i \neq j} \exp \left(-i k_{i} x_{i}\right), \\
P(k) & =a^{d} \sum_{x} P_{x} \prod_{i=1}^{d} \exp \left(-i k_{i} x_{i}\right),
\end{aligned}
$$

where $k \in[-\pi / a, \pi / a]^{d}$. We note that given the geometry of Fig. 1 , if $P_{x}$ lie at points $x$ whose coordinates are integers, then $V_{j, x}$ will lie at a point $x$ whose $j$-th coordinate is a half integer. Also, as before, it is possible to extend the lattice fields to momenta outside $[-\pi / a, \pi / a]$ by letting

$$
\begin{aligned}
V_{j}\left(k+2 \pi l_{i} \hat{i} / a\right) & =V_{j}(k), i \neq j \\
V_{j}\left(k+2 \pi l_{j} \hat{j} / a\right) & =(-1)^{l_{j}} V_{j}(k), \\
P\left(k+2 \pi l_{i} \hat{i} / a\right) & =P(k), \text { for all } i .
\end{aligned}
$$

We are thus ready to go to Fourier space where the above eqations take the form

$$
\begin{aligned}
& -k^{2} v_{j}(k)-\frac{i}{\nu \rho} k_{j} p(k)+\Pi_{j}(k) \eta_{j}(k)=0 \\
& V_{j}(k)-\sum_{l \in \mathbf{Z}^{d}} v_{j}(k+2 \pi l / a) \Pi_{j}(k+2 \pi l / a)(-1)^{l_{j}}-a^{2} \alpha \eta_{j}=0 \\
& \frac{i}{\nu \rho} k_{j} v_{j}(k)+\Pi(k) \eta(k)=0 \\
& P(k)-\sum_{l \in \mathbf{Z}^{d}} p(k+2 \pi l / a) \Pi(k+2 \pi l / a)-a^{2} \beta \eta(k)=0 .
\end{aligned}
$$

Solving for the continuum fields we obtain

$$
\begin{aligned}
& p(k)=-(\nu \rho)^{2} \Pi(k) \eta(k)-\frac{i \nu \rho}{k^{2}} k_{j} \Pi_{j}(k) \eta_{j}(k) \\
& v_{i}(k)=\frac{i \nu \rho}{k^{2}} k_{i} \Pi(k) \eta(k)-\frac{k_{i} k_{j}-k^{2} \delta_{i j}}{k^{4}} \Pi_{j}(k) \eta_{j}(k) .
\end{aligned}
$$

Reinserting these back into eq. 6.12) and eq.(6.14) gives equations of motion for the lattice fields and the Lagrange multiplier fields

$$
\begin{aligned}
& V_{i}(k)-i \nu \rho \omega_{i}(k) \eta(k)+\omega_{i j}(k) \eta_{j}(k)=0 \\
& P(k)+(\nu \rho)^{2} \omega(k) \eta(k)+i \nu \rho \omega_{j}(k) \eta_{j}(k)=0 .
\end{aligned}
$$


Here we have introduced the functions

$$
\begin{aligned}
& \omega(k)=\sum_{l \in \mathbf{Z}^{d}} \Pi(k+2 \pi l / a)^{2}+\beta=1+\beta \\
& \omega_{i}(k)=\sum_{l \in \mathbf{Z}^{d}} \frac{k_{i}+2 \pi l_{i} / a}{(k+2 \pi l / a)^{2}} \Pi_{i}(k+2 \pi l / a) \Pi(k+2 \pi l / a)(-1)^{l_{i}} \\
& \omega_{i j}(k)^{-1}=\sum_{l \in \mathbf{Z}^{d}} \frac{\left(k_{i}+2 \pi l_{i} / a\right)\left(k_{j}+2 \pi l_{j} / a\right)-(k+2 \pi l / a)^{2} \delta_{i j}}{(k+2 \pi l / a)^{4}} \\
& \times \Pi_{i}(k+2 \pi l / a) \Pi_{j}(k+2 \pi l / a)(-1)^{l_{i}+l_{j}}+a^{2} \alpha .
\end{aligned}
$$

We can rewrite the above equations in a simple fashion by letting $V_{0}=P$ and $\eta_{0}=\eta$

$$
V_{\mu}=\Omega_{\mu \nu}^{-1} \eta_{\nu}
$$

where $\Omega_{\mu \nu}$ is defined through the above relations. Finally, we solve for the Lagrange multiplier fields and set them equal to zero to obtain the equations of motion for the lattice fields

$$
\Omega_{\mu \nu} V_{\nu}=0 \text {. }
$$

We must now optimize for locality. As before, we go to $d=1$ and choose $\alpha$ and $\beta$ so that the equations of motion take an ultralocal form. In one dimension eq.(6.22) becomes

$$
\frac{1}{(1+\beta) \alpha-1 /(2 \sin (k / 2))^{2}}\left(\begin{array}{cc}
\alpha & i /(2 \sin (k / 2)) \\
-i /(2 \sin (k / 2) & 1+\beta
\end{array}\right)\left(\begin{array}{l}
P \\
V
\end{array}\right)=0 .
$$

Setting $\alpha=0$ and $\beta=0$, we obtain familiar ultralocal equations

$$
\begin{aligned}
& 2 \sin (k / 2) V(k)=0 \\
& 2 i \sin (k / 2) P(k)=(2 \sin (k / 2))^{2} V(k) .
\end{aligned}
$$

Having solved this simplified version of the Navier-Stokes equations we proceed to add the nonlinear term.

\section{Perturbative treatment of the nonlinear term}

Since the nonlinear term of the Navier-Stokes equation is quadratic in the velocity, it is possible to solve the lattice equations of motion only perturbatively. Here we demonstrate how to do this for the first order correction. We begin by introducing the modified version of eq.(6.11), now with the new term

$$
-\frac{i}{\nu} v_{j}(k) k_{j} v_{i}(k)-k^{2} v_{i}(k)-\frac{i}{\nu \rho} k_{i} v_{i}(k)+\Pi_{j}(k) \eta_{j}(k)=0 .
$$


Because we are only intersted in the first order correction we need only keep terms in the nonlinear contribution that are quadratic in the Lagrange multiplier fields. Thus, for the nonlinear term, it is sufficient to express the continuum fields as linear functions of the Lagrange fields. We make the following ansatz for $p^{(2)}$ and $v_{i}^{(2)}$, the second order contributions:

$$
\begin{aligned}
& p^{(2)}=A \eta(k)^{2}+\eta(k) B_{j}(k) \eta_{j}(k)+\eta_{i}(k) C_{i j}(k) \eta_{j}(k) \\
& v_{i}^{(2)}=D_{i}(k) \eta(k)^{2}+\eta(k) E_{i j} \eta_{j}(k)+F_{i j k} \eta_{j}(k) \eta_{k}(k) .
\end{aligned}
$$

Then $p^{(2)}$ and $v_{i}^{(2)}$ must obey the following equations,

$$
\begin{aligned}
& \frac{i \nu \rho^{2}}{k^{2}} k_{i} \Pi(k)^{2} \eta^{2}-\eta \frac{\rho\left(k_{i} k_{j}-k^{2} \delta_{i j}\right)}{k^{4}} \Pi(k) \Pi_{j}(k) \eta_{j}-k^{2} v_{i}^{(2)}-\frac{i}{\nu \rho} k_{i} p^{(2)}=0 .(7.4 \\
& k_{i} v_{i}^{(2)}(k)=0 .
\end{aligned}
$$

Solving them, we obtain

$$
\begin{aligned}
& p^{(2)}(k)=\nu^{2} \rho^{3} \frac{\Pi(k)^{2}}{k^{2}} \eta(k)^{2} \\
& v_{i}^{(2)}(k)=\rho \eta(k) \frac{k_{i} k_{j}-k^{2} \delta_{i j}}{k^{6}} \Pi_{j}(k) \Pi(k) \eta_{j}(k) .
\end{aligned}
$$

This procedure can now be repeated for the lattice fields. We make the ansatz,

$$
\eta_{\lambda}^{(2)}(k)=V_{\mu}(k) A_{\mu \nu}^{\lambda}(k) V_{\nu}(k),
$$

where again $V_{0}=P$. Keeping only the second order terms, yields

$$
\begin{aligned}
& -i \nu \rho \omega_{i} V_{\mu} A_{\mu \nu}^{0} V_{\nu}+\omega_{i j}(k) V_{\mu} A_{\mu \nu}^{j} V_{\nu}-\rho V_{\mu} \Omega_{\mu 0}^{T} S_{i j} \Omega_{j \nu} V_{\nu}=0 \\
& (\nu \rho)^{2} \omega V_{\mu} A_{\mu \nu}^{0} V_{\nu}+i \nu \rho \omega_{j} V_{\mu} A_{\mu \nu}^{j} V_{\nu}-\nu^{2} \rho^{3} V_{\mu} \Omega_{\mu 0}^{T} S \Omega_{0 \nu} V_{\nu}=0 .
\end{aligned}
$$

Here, the following functions have been introduced

$$
\begin{aligned}
& S(k)=\sum_{l \in \mathbf{Z}^{d}} \frac{1}{(k+2 \pi l / a)^{2}} \Pi_{i}(k+2 \pi l / a)^{3} \\
& S_{i j}(k)=\sum_{l \in \mathbf{Z}^{d}} \frac{\left(k_{i}+2 \pi l_{i} / a\right)\left(k_{j}+2 \pi l_{j} / a\right)-(k+2 \pi l / a)^{2} \delta_{i j}}{(k+2 \pi l / a)^{6}} \\
& \times \Pi_{i}(k+2 \pi l / a) \Pi_{j}(k+2 \pi l / a) \Pi(k+2 \pi l / a)(-1)^{l_{i}+l_{j}} .
\end{aligned}
$$

We rewrite the above equations in a more compact form

$$
\Omega_{\sigma \lambda}^{-1} A_{\mu \nu}^{\lambda}=M_{\mu \nu}^{\sigma}
$$

This, finally gives the first order nonlinear equation of motion

$$
\Omega_{\mu \nu} V_{\nu}+\Omega_{\mu \nu} V_{\lambda} M_{\lambda \sigma}^{\nu} V_{\sigma}=0
$$

In principal this procedure could be repeated to obtain non-linear lattice equations up to any order in the lattice fields. 


\section{Conclusions}

In this paper we have performed the first steps towards a perfect discretization of the Navier Stokes equations. The essential idea is to define coarse grained variables by avering the continuum pressure and velocity fields over appropriate spatial regions. The corresponding averaged field variables naturally live on a lattice. In the cases of Hagen-Poisseille flow and slow static flows in general we derived the exact equations of motion for the lattice variables, and hence a perfect discretization of the corresponding continuum equations. The incorporation of boundary conditions is nontrivial, and has been done explicitly for Hagen-Poisseille flow. For practical applications it is essential that the perfectly discretized equations of motion are very local (although in general not ultralocal). In particular, the lattice couplings to distant variables decay exponentially, and one can safely restrict oneself to a few nearby neighbors. In fact, we have performed numerical simulations using the perfectly discretized equations of motion for laminar flow in a channel with quadratic cross section and we have verified explicitly that an arbitrarily coarse lattice indeed gives continuum answers. Our method can be directly applied to more general geometries, although non-cubic lattices require nontrivial modifications. An important aspect of our construction are the so-called perfect fields. Indeed our method allows to interpolate the continuum fields from the lattice data. Hence, especially when we are working on a coarse lattice, we are not limited to lattice points.

Of course, a method like that would be most welcome in numerical simulations of more complicated fluid dynamics systems, especially in the case of turbulence. In these simulations the effects of a finite lattice spacing are the main source of systematic errors. We have made a first step in this direction by deriving the perfect equations of motion in the presence of the nonlinear term that gives rise to turbulence. However, we have included that term only to first order, while a perfect discretization of turbulent flows would require a treatment to all orders. This could be achieved numerically in analogy to [2] by solving a minimization problem on a multigrid. When one wants to simulate turbulence one should not restrict oneself to static flows. Then the definition of the averaged lattice variables should be generalized. In particular, one should also average pressure and velocity in time. In addition, one may want to simulate compressible fluids, which would bring in an averaged density variable. Our techniques are still applicable to these cases, but the actual implementation is nontrivial.

In conclusion, we have proposed a renormalization group treatment of problems in fluid dynamics. The renormalization group implies that perfect discretizations of the continuum Navier-Stokes equations exist. The perfectly discretized equations of motion have been constructed explicitly for slow static flows, and have been optimized for locality. This is essential in practical applications. The renormalization group offers a systematic way of treating small scale effects in fluid dynamics. Realizing this program for the full Navier-Stokes problem requires a lot of work. Still, 
the first steps that we have taken, have led to promising results.

\section{References}

[1] K. Wilson and J. Kogut, Phys. Rep. C12 (1974) 75.

[2] P. Hasenfratz and F. Niedermayer, Nucl. Phys. B414 (1994) 785.

[3] U.-J. Wiese, Phys. Lett. B315 (1993) 417.

[4] W. Bietenholz, E. Focht and U.-J. Wiese, Nucl. Phys. B436 (1995) 393.

[5] T. DeGrand, A. Hasenfratz, P. Hasenfratz and F. Niedermayer, Nucl. Phys. B454 (1995) 587; 615.

[6] W. Bietenholz and U.-J. Wiese, hep-lat/9510026, to appear in Nucl. Phys. B.

[7] K. G. Wilson, in New Pathways in High Energy Physics II, ed. A. Perlmutter (Plenum, New York 1976) 243.

[8] T. L. Bell and K. Wilson, Phys. Rev. B11 (1975) 3431. 